\title{
Suicidal ideation among the hypertensive individuals in Shandong, China: a path analysis
}

Dandan Ge ${ }^{1+}$, Xinyi Zhang ${ }^{1+}$, Xiaolei Guo ${ }^{2}$, Jie $\mathrm{Chu}^{3}$, Long Sun ${ }^{1}$ and Chengchao Zhou ${ }^{1,4^{*}}$

\begin{abstract}
Background: Although massive studies have explored the risk factors of suicidal ideation (SI), the prevalence of SI and its associated factors in the hypertensive individuals are largely unknown. This study aims to investigate the factors associated with SI in the hypertensive individuals.

Methods: Three thousand nine hundred eleven hypertensive individuals in Shandong, China were included in the analysis. SI was assessed by using a question from the NCS (National Comorbidity Survey). We used binary logistic regression analysis to explore the factors associated with $\mathrm{SI}$, and path analysis to test the direct and indirect relationships between associated factors and SI among hypertensive patients.
\end{abstract}

Results: The prevalence of SI in the hypertensive individual was19.6\%.

Psychological distress had the greatest direct $(\beta=0.640, p$-value $<0.01)$ and total effect $(\beta=0.640, p$-value $<0.01)$ on SI. Other factors including comorbidity $(\beta=0.090, p$-value $<0.01)$, gender $(\beta=0.088, p$-value $<0.01)$, marital status $(\beta=-0.037, p$-value $<0.01)$, economic status $(\beta=-0.106, p$-value $<0.01)$, residence $(\beta=-0.050, p$-value $<0.01)$, alcohol use $(\beta=0.011, p$-value $<0.01)$, exercise $(\beta=-0.114$, $p$-value $<0.01)$, hospitalization $(\beta=0.041, p$-value $<0.01$ ) only had indirect effects on SI. Psychological distress was a mediator between SI and those variables.

Conclusion: A significant mediation effect of psychological distress on the associations between SI and some associated factors (i.e., economic status, comorbidity) was demonstrated.

Keywords: Hypertension, Suicidal ideation, Psychological distress, Path analysis

\section{Background}

A global brief on hypertension by WHO reported that, the prevalence of hypertension in adults aged 25 and above was about $40 \%$ around the world in 2008, rising from 600 million in 1980 to 1 billion in 2008 during the past 28 years [1]. Similarly, this prevalence among the adults aged 18 and above in China has risen from 18.8\% in 2002 to $25.2 \%$ in 2015 , Presently, there are more than 200 million hypertensive patients in China [2]. Moreover, hypertension is a common cardiovascular and

\footnotetext{
*Correspondence: zhouchengchao@sdu.edu.cn

${ }^{\dagger}$ Dandan Ge and Xinyi Zhang have an equal contribution and are the co-first authors.

${ }^{1}$ School of Public Health, Shandong University, Jinan 250012, China

${ }^{4}$ School of Public Health, NHC Key Lab of Health Economics and Policy

Research, Shandong University, Jinan 250012, China

Full list of author information is available at the end of the article
}

cerebrovascular disease which plays a major role in the development of cerebrovascular disease, cardiac and renal failure [3]. It was estimated that, 2.5 million Chinese adults died from hypertension and its comorbidities, accounting for $28 \%$ of all death in 2013 [4]. Hypertension is the leading risk factor for cardiovascular disease and premature death, which is an important public health problem in China $[5,6]$.

Suicidal ideation (SI) is a significant risk factor for suicidal attempt and suicide, which is a momentous part and inevitable stage of suicidal behavior [7, 8]. Many previous studies have shown that the SI was associated with chronic physical conditions (asthma, rheumatoid arthritis and hypertension) [9-11]. Some observed relationship between hypertension and mental health showed that hypertensive patients found to manifest symptoms of depression and stress, which were strongly

(C) The Author(s). 2019 Open Access This article is distributed under the terms of the Creative Commons Attribution 4.0 International License (http://creativecommons.org/licenses/by/4.0/), which permits unrestricted use, distribution, and 
related to SI [12]. In addition, a study based on DEPSCREEN-INFO clinical trial pointed out that hypertension was a clinical vulnerability of SI [11].

To the best of our knowledge, numerous studies have explored the risk factors of SI [11-13]. However, few studies have explored the SI and its associated factors in the hypertensive individuals, and no studies have examined the direct and indirect relationship between associated factors and SI among the hypertensive patients in China.

The current study aims to identify the risk factors of SI among the hypertensive patients. Firstly, this study will analyze the prevalence of the SI among the hypertensive patients. Secondly, this study will compare the SI across different subgroups of the hypertensive patients. Finally, using a path analysis method, this study will explore the direct and indirect relationship between associated factors and SI among the hypertensive patients in China.

\section{Methods}

\section{Subjects}

This study was conducted in Shandong Province, from August to October, 2016. Shandong ranks the second in the number of population in China. The prevalence of hypertension in the adults aged 18 to 69 in 2013 was $27.9 \%$, which was significantly higher than the average level in the whole country [2]. A three-stage stratified cluster sampling method was used to select the participants. First, according to the proportion of urban to rural residents (1 to 2) and Gross Domestic Product (GDP) per capita in Shandong in 2015, we selected two urban districts (one upper the medium GDP level, Fushan, and the other below the medium GDP level, Weicheng) and four rural counties (one upper the medium level, Rushan, two at the medium level, Yiyuan, and Gaotang, and one below the medium level, Liangshan) as study sites. Second, according to GDP per capita, all of the sub-districts and townships in each selected urban districts and rural counties were divided into three levels. For each level, one sub-district and one township were randomly selected. Third, we randomly selected two communities or villages with more than 1000 permanent residents from each of the selected subdistricts and townships. More details about data collection methods were reported in our previous studies [14].

The criterion for hypertension was systolic blood pressure $(\mathrm{SBP}) \geq 140 \mathrm{~mm}$ HG $(1 \mathrm{~mm}$ HG $=0.133 \mathrm{kPa})$ and diastolic blood pressure (DBP) $\geq 90 \mathrm{~mm}$ HG, following the Chinese guideline for the management of hypertension in 2005 [15]. In addition, the standards for hypertension diagnosis have been further strengthened since 2010 [16]. In this study, all hypertensive patients who have already been diagnosed as hypertension by medical professionals of different-level medical institutions and registered in the sample community or village chronic case management system were included. We excluded those who were unable to communicate with the interviewers properly. In total, 3911 hypertensive patients were included in the current study.

\section{Data collection}

The healthcare practitioners in sample community health stations or village clinics were responsible for recruiting the hypertensive patients in the list of chronic case management system. About 1 week before the normal survey, the hypertensive patients were informed of the purpose and time of the interview by the healthcare practitioners and then signed the willingness for the participation in the survey.

All the subjects were interviewed face-to-face using a standard structured questionnaire by trained postgraduate students from Shandong University School of Public Health. Before the interview, the written consent were obtained from each of the participants. To achieve the purposes of this study, completed questionnaires were subject to stringent quality assurance.

\section{Variables \\ Social demographic characteristics}

The social demographic characteristics of the participants included age, gender, education, marital status, residence, and economic status. When measuring "Economic status", we used a question of "How much was the total income of your household in the past year (Urban households were disposable income, rural households were net income)?" It was divided into four same group distance: 25\% (Quartile 1,Q1), 50\% (Quartile 2, Q2),75\% (Quartile 3,Q3), and 100\% (Quartile 4,Q4), and Q1 was the poorest, and Q4 was the richest.

\section{Health behaviors}

The behavioral variables were assessed using smoking, alcohol use, and exercise. These characteristics were defined as follows: smoking (current smokers and the cumulative quantity is more than 100 cigarettes before the survey, yes vs. no) [17-19], alcohol use (drinking on any day in the past 30 days, yes vs. no) [20, 21], exercise $(<1,1-2,>=3$ times a week $)$.

\section{Variables associated with hypertensive health}

Variables associated with hypertensive health were obtained through types of anti-hypertension drugs in the previous 2 weeks $(0,1, \geq 2)$, hospitalization due to hypertension in the past year (yes vs. no), and duration of hypertension $(<5,5-, 15-, 20+$ years $)$. 


\section{Psychological distress (K10)}

This study applied 10-item Kessler Psychological Distress Scale which was composed of 10 items to evaluate the status of psychology distress, mainly focusing on depression and anxiety during the past 30 days. This scale was used widely in various countries for screening psychological distress $[22,23]$. Each item includes five dimensions from "none of the time" to "all of the time", and is scored from 1 to 5 . A total score of 10 to 19 was coded into1, which was suggestive of lower risk of suffering from mental illness; 20 to 24 points was coded into 2, which was indicative of low risk of a mental illness; 25 to 29 points was coded into 3 , which was suggestive of relatively high risk of psychiatric disorders, and 30 to 50 was coded into 4, which was indicative of higher risk of mental disorders [24]. The Cronbach's $\alpha$ of the K10 in this study was 0.89 .

\section{Suicidal ideation}

A question of "Have you ever seriously considered committing suicide?" was used to measure SI in this study. If the answer was 'yes', SI was coded as ' 1 ', and if the answer was 'no', SI was coded as '0'. The question comes from the baseline NCS (National Comorbidity Survey), which has been widely used in many previous studies with high reliability and validity $[25,26]$.

\section{Statistical analysis}

All data were analyzed by using Mplus7.0 and SPSS 22.0.We used percentages to describe the demographics and Chi-square test to compare the prevalence of SI across different subgroups of the hypertensive patients. Binary logistic regression models were employed to explore the factors associated with SI. We also used a path analysis model to identify the direct and indirect effect of associated factors on SI. The statistical significance was evaluated at the level of $5 \%$. In order to avoid potential alpha inflation, we adjusted the significance level by the number of multiple tests in the multiple models.

\section{Results}

Of the 3911 subjects, 767 (19.6\%) had SI. The patients with comorbidities had a significantly higher prevalence of SI (25.2\%) compared with those without comorbidities (16.8\%). The prevalence of SI among singles was $27.0 \%$, which was higher than those with a couple $(18.0 \%)$. The hypertensive individuals from rural areas had a higher prevalence of SI $(21.6 \%)$ than those from urban areas (12.0\%). The current alcohol users had a higher SI rate (21.3\%) than their counterparts (11.3\%). Those patients who had hospitalization had a significantly higher prevalence of SI (26.9\%) than those without hospitalization due to hypertension in the previous year (18.5\%). As for the psychological well-being, the patients with higher level of K10 were more likely to experience SI (See the Table 1).

We presented the results of this study to explore the relationships between related variables and SI using two models. In model 1 , we included variables except K10. The model showed that the variables including comorbidity, gender, marital status, economic status, residence, alcohol use, exercise, and hospitalization were significantly associated with SI. When we included K10 in Model 2, economic status and marital status were still significantly associated with SI (See the Table 2).

We used a path analysis model to further explore the relationship between SI and related variables in hypertensive patients. As shown in Table 3 and Fig. 1, nine specified factors (comorbidity, gender, marital status, economic status, residence, alcohol use, exercise, hospitalization and K10) included in the model. Standardized total effects of the greatest absolute value was K10 (0.640), followed by exercise $(-0.124)$, economic status $(-0.112)$. We found that $\mathrm{K} 10$ had both direct impact on SI, and the other eight variables was mediated by K10 towards SI. Goodness of fit indices was employed to measure the model fit in this study. In this model, TLI (tucker-lewis index) had a value of 1.00, CFI (comparative fit index) value 0.99 , and a root mean square error of approximation (RMSEA) value 0.000 . The overall variables accounted for $44.1 \%$ of the variance in SI. All of these indexes have confirmed the path model.

\section{Discussion}

Our study provided for the first time a real profile of SI among the hypertensive individuals in China. In this study, we found that the prevalence of lifetime SI among the hypertensive was $19.6 \%$. It was higher than the reported incidence of SI (13.5\%) in adults aged from 15 to 54 by Kessler et al. in 1999 [27]. It was also much higher than the reported SI rate in rural and urban residents in Beijing (2.3\%), older adults aged 60 and above (4.2\%), community-dwelling elders in Taiwan (6.1\%), and medical college students (12.9\%) in China [28-31]. When compared with those with chronic diseases, we also found that it was higher than the reported SI rate of $8.7 \%$ in the individuals with asthma and $12.0 \%$ in the patients with COPD (chronic obstructive pulmonary disease) in United States [32, 33]. This was also higher than the reported SI rate of $11 \%$ in the hospital outpatient with rheumatoid arthritis in United Kingdom and Chinese patients with COPD (15.4\%) [10,34]. The prevalence of SI in the current study was lower than what was reported in patients with asthma $(21.4 \%)$ in Korea, patients with AIDS during their lifetime (27.2\%) in Changsha city, and also patients with ovarian cancer (30.3\%) in Hunan Province, China [9, 35, 36]. Even though the SI rate in the current study is a little lower 
Table 1 Factors associated with suicidal ideation among the hypertensive patients in Shandong, China, 2016

\begin{tabular}{lllll}
\hline Characteristics & Total $n(\%)$ & \multicolumn{2}{l}{ Suicidal Ideation } & $P$ \\
\cline { 2 - 4 } & & Yes (\%) & No (\%) & \\
\hline Observation & 3911 & $767(19.6)$ & $3144(80.4)$ & \\
Comorbidity & & & & $\mathbf{0 . 0 0 0}$ \\
$\quad$ Yes & $1326(33.9)$ & $334(25.2)$ & $992(74.8)$ & \\
No & $2585(66.1)$ & $433(16.8)$ & $2152(83.2)$ &
\end{tabular}

Gender

Male

Female

Age

$<60$

$\geq 60$

Marital status

Single
Married

Education

$\begin{array}{llll}\text { Illiteracy or semiliterate } & 1502(38.4) & 349(23.2) & 1153(76.8) \\ \text { Primary school } & 1363(34.9) & 272(20.0) & 1091(80.0) \\ \begin{array}{l}\text { Junior school } \\ \text { Senior school or above }\end{array} & 750(19.2) & 112(14.9) & 638(85.1) \\ \text { Economic status }{ }^{b} & & 34(11.5) & 262(88.5) \\ \text { Q1 } & 982(25.1) & 250(25.5) & 732(74.5) \\ \text { Q2 } & 976(25.0) & 220(22.5) & 756(77.5) \\ \text { Q3 } & 977(25.0) & 187(19.1) & 790(80.9) \\ \text { Q4 } & 976(25.0) & 110(11.3) & 866(88.7)\end{array}$

Residence

$\begin{array}{clll}\text { Rural } & 3113(79.6) & 671(21.6) & 2442(78.4) \\ \text { Urban } & 798(20.4) & 96(12.0) & 702(88.0) \\ \text { Smoking } & & & \\ \text { No } & 2987(76.4) & 647(21.7) & 2340(78.3) \\ \text { Yes } & 924(23.6) & 120(13.0) & 804(87.0)\end{array}$

Alcohol use

$$
\begin{aligned}
& \text { No } \\
& \text { Yes }
\end{aligned}
$$

Exercise (time/a week)

$<1$
$1-2$
$\geq 3$

Number of medicine

$$
\begin{aligned}
& 0 \\
& 1
\end{aligned}
$$$$
\geq 2
$$

0.000

0.000

0.000
$1433(36.6) \quad 202(14.1) \quad 1231(85.9)$

$2478(63.4) \quad 565(22.8) \quad 1913(77.2)$

$847(21.7) \quad 155(18.3) \quad 692(81.7)$

$3064(78.3) \quad 612(20.0) \quad 2452(80.0)$

$686(17.5) \quad 185(27.0) \quad 501(73.0)$

$3225(82.5) \quad 582(18.0) \quad 2643(82.0)$

0.000

Hospitalization

$$
\text { No }
$$

Yes

$\mathrm{K} 10^{c}$

1

0.000

2

3

4
Table 1 Factors associated with suicidal ideation among the hypertensive patients in Shandong, China, 2016 (Continued)

\begin{tabular}{lllll}
\hline Characteristics & Total n (\%) & \multicolumn{2}{l}{ Suicidal Ideation } & $P$ \\
\cline { 3 - 4 } & & Yes (\%) & No (\%) & \\
\hline $\begin{array}{lllll}\text { Duration } \\
<5\end{array}$ & $1230(31.4)$ & $221(18.0)$ & $1009(82.0)$ & \\
$5-$ & $1234(31.6)$ & $239(19.4)$ & $995(80.6)$ & \\
$15-$ & $531(13.6)$ & $115(21.7)$ & $416(78.3)$ & \\
$20+$ & $916(23.4)$ & $192(21.0)$ & $724(79.0)$ & \\
Hospitalization & & & & $\mathbf{0 . 0 0 0}$ \\
No & $3371(86.2)$ & $622(18.5)$ & $2749(81.5)$ & \\
Yes & $540(13.8)$ & $145(26.9)$ & $395(73.1)$ & \\
K10 & & & & $\mathbf{0 . 0 0 0}$ \\
1 & $2358(60.3)$ & $144(6.1)$ & $2214(93.9)$ & \\
2 & $624(16.0)$ & $164(26.3)$ & $460(73.7)$ & \\
3 & $499(12.8)$ & $205(41.1)$ & $294(58.9)$ & \\
4 & $430(11.0)$ & $254(59.1)$ & $176(40.9)$ & \\
\hline
\end{tabular}

P-values indicate statistical significance at $5 \%$ level

${ }^{\mathrm{a}}$ Singles include those who are unmarried $(1.7 \%)$, divorced $(0.3 \%)$, widowed (15.5\%)

${ }^{b}$ Quartile $1(\mathrm{Q} 1)$ is the poorest and Quartile 4(Q4) is the richest

'K10 means 10-item Kessler Psychological Distress Scale:1 was suggestive of lower risk of suffering from mental illness, 2 was indicative of low risk of a mental illness, 3 was suggestive of relatively high risk of psychiatric disorders, and 4 was indicative of higher risk of mental disorders

than those in the patients with AIDS or ovarian cancer, it is higher than those in general population and those patients with some other chronic diseases, which indicates that hypertension might be a serious type of chronic diseases that increase the risk of SI.

In this study, we found the psychological distress had the most potent effect (0.640) and direct impact (0.640) on SI. This finding was similar to the previous studies which indicated that psychological distress was the primary determinant of SI [37, 38]. Many studies have demonstrated that those who with high-level psychological distress would be more likely to have SI and suicidal plan [39-41]. Some studies showed that hypertension and psychological distress act together in a vicious cycle, and can sometimes lead to SI [42, 43]. Given the relationship between hypertension and psychological distress, we should develop interventions to decrease the prevalence of SI [44].

This study demonstrated that both hospitalization and comorbidity had indirect effect on SI mediated by psychological distress. Some studies revealed that hypertensive individuals with hospital admission during the previous year, or with comorbidities tend to experience mental illness and SI $[45,46]$. A study in elderly patients with hypertension showed a high level of depression, and comorbidity is the primary influencing factor for depression [47]. Another study about the causes of SI among the elderly indicated that the seniors suffering 
Table 2 Multivariate logistic regression models for factors associated with suicidal ideation among the hypertensive patients in Shandong, China, 2016

\begin{tabular}{|c|c|c|c|c|c|c|c|c|}
\hline \multirow[t]{2}{*}{ Characteristics } & \multicolumn{3}{|c|}{ Model 1} & \multicolumn{5}{|c|}{ Model 2} \\
\hline & $\mathrm{OR}$ & $95 \% C$ & & $P$-value & OR & $95 \% \mathrm{Cl}$ & & $P$-value \\
\hline \multicolumn{9}{|l|}{ Comorbidity } \\
\hline No & 1.00 & & & & 1.00 & & & \\
\hline Yes & 1.41 & 1.18 & 1.69 & 0.000 & 1.06 & 0.87 & 1.30 & 0.542 \\
\hline \multicolumn{9}{|l|}{ Gender } \\
\hline Male & 1.00 & & & & 1.00 & & & \\
\hline Female & 1.32 & 1.03 & 1.69 & 0.028 & 1.03 & 0.79 & 1.36 & 0.820 \\
\hline \multicolumn{9}{|l|}{ Marital status } \\
\hline Single $e^{a}$ & 1.00 & & & & 1.00 & & & \\
\hline Married & 0.70 & 0.57 & 0.87 & 0.001 & 0.79 & 0.62 & 0.99 & 0.043 \\
\hline \multicolumn{9}{|l|}{ Education } \\
\hline Illiteracy or semiliterate & 1.00 & & & & 1.00 & & & \\
\hline Primary school & 1.09 & 0.90 & 1.32 & 0.374 & 1.19 & 0.96 & 1.47 & 0.113 \\
\hline Junior school & 0.95 & 0.73 & 1.22 & 0.672 & 1.05 & 0.79 & 1.39 & 0.737 \\
\hline Senior school or above & 0.85 & 0.57 & 1.28 & 0.442 & 0.89 & 0.57 & 1.40 & 0.625 \\
\hline \multicolumn{9}{|l|}{ Economic status $^{\text {b }}$} \\
\hline Q4 & 1.00 & & & & 1.00 & & & \\
\hline Q1 & 1.85 & 1.39 & 2.46 & 0.000 & 1.23 & 0.90 & 1.68 & 0.199 \\
\hline Q2 & 1.81 & 1.37 & 2.39 & 0.000 & 1.32 & 0.98 & 1.79 & 0.069 \\
\hline Q3 & 1.56 & 1.19 & 2.06 & 0.001 & 1.35 & 1.00 & 1.82 & 0.047 \\
\hline \multicolumn{9}{|l|}{ Residence } \\
\hline Urban & 1.00 & & & & 1.00 & & & \\
\hline Rural & 1.37 & 1.05 & 1.80 & 0.021 & 1.25 & 0.93 & 1.68 & 0.134 \\
\hline \multicolumn{9}{|l|}{ Smoking } \\
\hline No & 1.00 & & & & 1.00 & & & \\
\hline Yes & 0.78 & 0.58 & 1.03 & 0.079 & 0.74 & 0.54 & 1.01 & 0.055 \\
\hline \multicolumn{9}{|l|}{ Alcohol use } \\
\hline No & 1.00 & & & & 1.00 & & & \\
\hline Yes & 0.72 & 0.53 & 0.97 & 0.030 & 0.91 & 0.66 & 1.26 & 0.567 \\
\hline \multicolumn{9}{|l|}{ Exercise (time/a week) } \\
\hline$<1$ & 1.00 & & & & 1.00 & & & \\
\hline $1-2$ & 0.74 & 0.52 & 1.06 & 0.096 & 1.17 & 0.44 & 1.26 & 0.439 \\
\hline$\geq 3$ & 0.57 & 0.48 & 0.67 & 0.000 & 0.88 & 0.73 & 1.07 & 0.198 \\
\hline \multicolumn{9}{|l|}{ Hospitalization } \\
\hline No & 1.00 & & & & 1.00 & & & \\
\hline Yes & 1.38 & 1.09 & 1.74 & 0.007 & 1.18 & 0.91 & 1.53 & 0.218 \\
\hline \multicolumn{9}{|l|}{$\mathrm{K} 10^{\mathrm{C}}$} \\
\hline 1 & & & & & 1.00 & & & \\
\hline 2 & & & & & 4.95 & 3.85 & 6.36 & 0.000 \\
\hline 3 & & & & & 9.34 & 7.25 & 12.03 & 0.000 \\
\hline 4 & & & & & 18.94 & 14.46 & 24.81 & 0.000 \\
\hline
\end{tabular}

Note: The $P$-values indicate statistical significance at $5 \%$ level

${ }^{\mathrm{a}}$ Singles include those who are unmarried $(1.7 \%)$, divorced $(0.3 \%)$, widowed $(15.5 \%)$

${ }^{b}$ Quartile $1(\mathrm{Q} 1)$ is the poorest and Quartile $4(\mathrm{Q} 4)$ is the richest

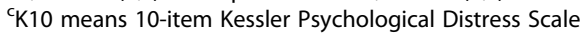


Table 3 Standardized effects on suicidal ideation from path analysis among the hypertensive patients in Shandong, China, 2016

\begin{tabular}{llll}
\hline & Total effect & Direct effect & Indirect effect \\
\hline Gender & $0.105^{* *}$ & 0.017 & $0.088^{* *}$ \\
Marital status & $-0.066^{*}$ & -0.030 & $-0.037^{* *}$ \\
Economic status & $-0.112^{* *}$ & -0.006 & $-0.106^{* *}$ \\
Residence & $-0.084^{*}$ & -0.034 & $-0.050^{* *}$ \\
Alcohol use & $0.071^{*}$ & 0.061 & $0.011^{* *}$ \\
Exercise & $-0.124^{* *}$ & -0.010 & $-0.114^{* *}$ \\
Hospitalization & $0.058^{*}$ & 0.017 & $0.041^{* *}$ \\
Comorbidity & $0.090^{* *}$ & -0.003 & $0.090^{* *}$ \\
K10 & $0.640^{* *}$ & $0.640^{* *}$ & - \\
\hline
\end{tabular}

Note: The total effects are the sum of the direct and indirect effects; : $P<0.05,{ }^{* *}: P<0.01$

${ }^{\mathrm{a}}$ K10 means 10-item Kessler Psychological Distress Scale

from disability due to long-term chronic diseases had a significant impact on psychological disorders and SI [48]. Placido et al. pointed out that the incidence of suicide was positively correlated with those with ischemic heart disease and stroke [49]. This finding indicated that psychological distress played an important meditating role in the association between hospitalization, comorbidity and SI among the hypertensive patients.

Negative direct and indirect relationships were detected between economic status and SI, which were similar to previous studies. Some studies have demonstrated that financial situation was an associated factor for SI mediated by mental illness [50, 51]. Some studies also found people with financial difficulties were more likely to experience psychological disorders and suicide
[52-54]. Similar to the previous studies, we also found marital status had an indirect effect on SI. Some studies demonstrated that the rate of SI in married patients was lower than that in as table married (unmarried, divorced, widowed) patients [55]. This might be due to the protection of social support from the family. Zhang et al. found that social support from family moderated the suicidal thoughts [56]. A study by Yang and Clum also found that mental illness was a mediator between social support and SI [57]. In this study, we also found gender had an indirect association with SI. A survey conducted by Feng et al. demonstrated that the female had a higher risk for SI than the male, which might be due to Chinese Confucianism [58]. In addition, our study showed that healthy lifestyles, including exercise and non-use of alcohol, had an adverse effect on SI, which was consistent with some previous studies $[59,60]$.

Interestingly, it was observed that all variables related to SI were mediated by psychological distress. The current study demonstrated a close and significant relationship between psychological distress and SI in hypertensive patients. A study conducted by Bruce et al. in the USA indicated that depression was the primary risk factor of SI, and an intervention of primary care can efficiently reduce the prevalence of SI in the depressive patients [61]. It was vital for the policymakers to develop interventions to regularly monitor the psychological well-being in the hypertensive individuals for early detection of psychological disorders, especially for those at-risk subgroups (e.g., those hypertensive patients with comorbidities, hospitalization use or poor socioeconomic status), so as to prevent the potential SI in the hypertensive individuals in China.

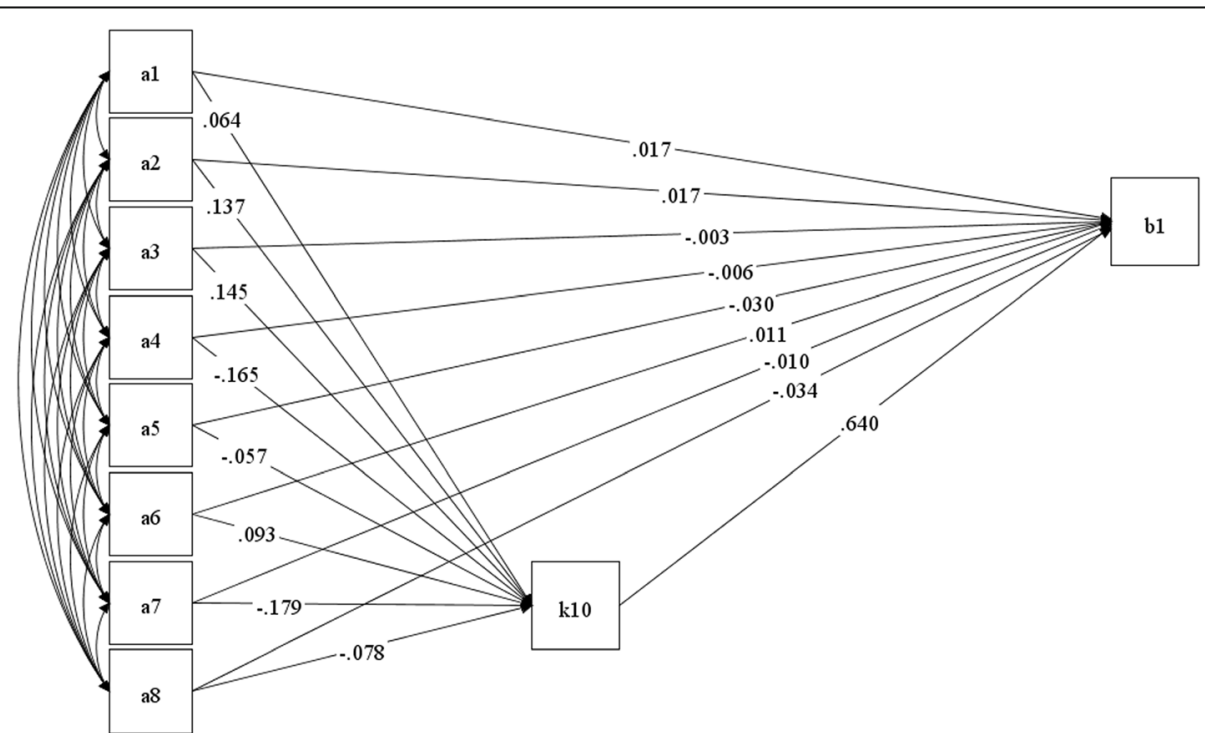

Fig. 1 Path analysis of suicidal ideation among the hypertensive individuals in Shandong ( $n=3911)$, China, 2016. k10: Psychological distress, a1: Hospitalization, a2: Gender, a3: Comorbidity, a4: Economic status, a5: Marital status, a6: Alcohol use, a7: Exercise, a8: Residence, b1: Suicidal ideation 
The current study is restricted to the hypertensive patients, the associations between identified factors and SI, and also the path model, might be somewhat specific for the hypertensive patients. In addition, the coefficients of the path analysis were high. Hence, future work will focus on examining whether the associations and path model can be generalized to other populations.

This study had some limitations. First, as a cross-sectional survey, the associations between identified factors and SI measured in this study could not be interpreted as causality. In addition, owing to the limitation of the cross-sectional study, we could only measure the prevalence of SI, but could not assess the incidence of the SI. Second, in this study, we just concentrated on the associated factors for SI, and gave no concerns about some other suicidal behaviors (e.g., plans, attempts), which would be remedied in the follow-up study. Third, the measurement of the SI mainly relied on one question and self-report information, which might result in inevitable bias. Fourth, the measurement of current smoking behavior was somewhat crude, and could not indicate the quantity, which would also be remedied in the follow-up study. Finally, some other possible confounding factors were not included in the analysis, which will be made up in the follow-up study.

\section{Conclusions}

Psychological distress had the strongest direct and total effect on SI. Factors including comorbidity, gender, marital status, economic status, residence, alcohol use, exercise, and hospitalization only had indirect effects on SI. A significant mediation effect of psychological distress on the associations between SI and aforementioned risk factors (i.e., comorbidity, gender) was demonstrated. These findings of the path analysis would improve our understanding of the mechanisms of the effect of different associated factors on SI in hypertensive individuals and facilitate selection of intervention strategies by targeting at the associated factors, so as to prevent subsequent suicidal behaviors.

\section{Abbreviations}

CFI: comparative fit index; COPD: chronic obstructive pulmonary disease; DBP: diastolic blood pressure; GDP: Gross Domestic Product; K10: 10-item Kessler Psychological Distress Scale; NCS: National Comorbidity Survey; RMSEA: root mean square error of approximation; SBP: systolic blood pressure; SI: Suicidal ideation; TLI: tucker-lewis index; WHO: World Health Organization

\section{Acknowledgements}

We especially thank Shandong Centre for Disease Control and Prevention, and its staff members, to give much support in the conduction of the field survey. We thank the officials of local health agencies and all participants and staff at the study sites for their cooperation. We wish to thank the reviewers for their insightful comments.

\section{Authors' contributions}

DDG and XYZ conceived the idea. XLG, JC and LS participated in data collection and analysis. CCZ assisted in reviewing the manuscript and addressing the statistical comments. All authors read and approved the final manuscript.

\section{Funding}

This study was supported by the National Science Foundation of China (71774104, 71473152 and 71003067), the China Medical Board (16-257), Cheeloo Youth Scholar Grant, and Shandong University (IFYT1810, 2012DX006). The funding bodies had no role in the design, data collection, analysis, interpretation of the data, and writing of this article.

\section{Availability of data and materials}

Please contact the corresponding author for data requests.

\section{Ethics approval and consent to participate}

This study was approved by Ethical Committee of Shandong University School of Public Health. Before the interview, the purpose of the study was explained to the participants and written informed consents were obtained from each of the participants.

\section{Consent for publication}

Not applicable.

\section{Competing interests}

The authors declare that they have no competing interests.

\section{Author details}

${ }^{1}$ School of Public Health, Shandong University, Jinan 250012, China.

${ }^{2}$ Shandong Center for Disease Control and Prevention, Jinan, China.

${ }^{3}$ Shandong Centre for Disease Control and Prevention, Jinan 250014, China.

${ }^{4}$ School of Public Health, NHC Key Lab of Health Economics and Policy

Research, Shandong University, Jinan 250012, China.

Received: 2 October 2018 Accepted: 22 August 2019

Published online: 02 September 2019

\section{References}

1. WHO, 2013. A global brief on Hypertension-Silent killer, global public health crisis.

2. National Health and Family Planning Commission of the People's Republic of China. 2015 report on Chinese resident's chronic disease and nutrition. Beijing: People's Medical Publishing House; 2015. in Chinese

3. Zhao M, Ji J. A review of suicide research in China. J Clin Psychiatry. 2002;12:161-3 in Chinese.

4. Li Y, Yang L, Wang $L$, et al. Burden of hypertension in China: a nationally representative survey of 174,621 adults. Int J Cardiol. 2017;227:516-23.

5. He J, Gu D, Wu X, et al. Major causes of death among men and women in China. N Engl J Med. 2005;353:1124-34.

6. He J, Gu D, Chen J, et al. Premature deaths attributable to blood pressure in China: a prospective cohort study. Lancet. 2009;374:1765-72.

7. Goldstein RB, Black DW, Nasrallah A, Winokur G. The prediction of suicide sensitivity, specificity and predictive value of a multivariate model applied to suicide among 1906 patients with affective disorders. Arch Gen Psychiatry. 1991;48:418-22.

8. Schwab JJ, Warheit GJ, Holzer CE. Suicidal ideation and behavior in a general population. Diseases of the Nervous System. 1972;33:745-8.

9. Chung JH, Kim S-H, Lee YW. Suicidal ideation and suicide attempts among asthma. Ann General Psychiatry. 2016;15:35.

10. Gareth J, Treharne, Lyons AC, Kitas GD. Suicidal ideation in patients with rheumatoid arthritis: Research may help identify patients at high risk. BMJ. 2000 Nov 18:321(7271):1290

11. Lehmann, M.; Kohlmann, S.; Gierk, B.; Murray, A. M. \& Löwe, B. (2018), 'Suicidal ideation in patients with coronary heart disease and hypertension: Baseline results from the DEPSCREEN-INFO clinical trial.', Clinical psychology \& psychotherapy.

12. Kretchy IA, Owusu-Daaku FT, Danquah SA. Mental health in hypertension: assessing symptoms of anxiety, depression and stress on anti-hypertensive medication adherence. Int J Ment Heal Syst. 2014;8(1):25. 
13. Kessler RC, Berglund P, Borges G, Nock M, Wang PS. Trends in Suicide Ideation, Plans, Gestures, and Attempts in the United States,1990-1992 to 2001-2003. American Medical Association. 2005;293:2487-95.

14. Jing Z, Chu J, Imam Syeda Z, et al. Catastrophic health expenditure among type 2 diabetes mellitus patients: A province-wide studyin Shandong, China. J Diabetes Investig. 2018 Jul 25. https://doi.org/10.1111/jdi.12901.

15. Guidelines for prevention and treatment of hypertension in China (2005 Version), 2006. People's Medical Publishing House, Beijing (in Chinese).

16. Guidelines for prevention and treatment of hypertension in China (revised version 2010). See http://www.360doc.com/content/16/0320/08/2 00041_543731387.shtml(Accessed 27 Apr 2018, in Chinese).

17. WHO. Guidelines for controlling and monitoring the tobacco epidemic. Geneva: World Health Organization; 1998

18. Jiang F, Li S, Pan L, Wang Q, Yang X, Zhang N, et al. Mediating effect of smoking abstinence self-efficacy on association between awareness of smoking hazard and successful smoking cessation. Chin J Epidemiol. 2015;36(10):1081-4 in Chinese

19. Zhou H, Ma Y, Peng J, Yin P, Liu X, Zhang D. Analysis on smoking, quit smoking, and passive smoking among permanent residents in Shenzhen. Chinese journal of social medicine. 2011;28(5):329-31 in Chinese.

20. Chen X, Unger JB, Palmer P, Weiner MD, Johnson CA, Wong MM, Austin G. Prior cigarette smoking initiation predicting current alcohol use: evidence for a gateway drugeffect among California adolescents from eleven ethnic groups. Addict Behav. 2002;27(5):799-817.

21. Jiang S, Chu J, Li C, Medina A, Hu Q, Liu J, Zhou C. Alcohol consumption is higher among left-behind Chinese children whose parents leave rural areas to work. Acta Paediatr. 2015;104(12):1298-304.

22. Victorian Population Health Survey, 2001. Melbourne: Department of Human Services, Victoria.

23. Kessler R, Mroczek D, 1992. An update of the development of mental health screening scales for the US national health interview study. Ann Arbort (MI): Survey research center of the institute for social research. University of Michigan.

24. Zhou C, Chu J, Wang T, Peng Q, He J. Reliability and validity of the Chinese version of 10 Kessler scales in the assessment of mental health status in Chinese population. Chinese Journal of Clinical Psychology. 2008;16:627-9 (in Chinese).

25. Xu H, Qin L, Wang J, Zhou L, Luo D, Hu M, et al. A cross-sectional study on risk factors and their interactions with suicidal ideation among the elderly in rural communities of Hunan, China. BMJ Open. 2016;6(4):e010914.

26. Lee S, Fung SC, Tsang A, Liu ZR, Huang YQ, He YL, et al. Lifetime prevalence of suicide ideation, plan, and attempt in metropolitan China. Acta Psychiatr Scand. 2007;116(6):429-37.

27. Kessler RC, Borges G, Walters EE. Prevalence of and risk factors for lifetime suicide attempts in the National Comorbidity Survey. Arch Gen Psychiatry. 1999;56:617-26.

28. Ma X, Xiang YT, Cai ZJ, Li SR, Xiang YQ, Guo HL, et al. Lifetime prevalence of suicidal ideation, suicide plans and attempts in rural and urban regions of Beijing, China. Royal Australian and New Zealand College of Psychiatrists. 2009:43:158-66

29. Qian Y, Sun L, Zhou C, Ge D, Zhang L. The association between suicidal ideation and sleep quality in elderly individuals: a cross-sectional study in Shandong, China. Psychiatry res. Jul 10;256:453-457. 2017. https://doi.org/1 0.1016/j.psychres.2017.07.017.

30. Chan HL, Liu CY, Chau YL, Chang CM. Prevalence and association of suicide ideation among Taiwanese elderly - A population-based cross-sectional study. Chang Gung Med J. 2011;34(2):197-204.

31. Zang $M$, Wang $X$, Wang $Y$, Huang $X$, Wang J. A systematic review on suicidal ideation and its influencing factors among the medical students in China. Chinese Journal of School Health. 2016;37(5):711-5 (in Chinese).

32. Chung J, Kim S, Yong W. Suicidal ideation and suicide attempts among asthma. Ann General Psychiatry. 2016;15(1):35.

33. Shemesh E, Annunziato RA, Rubinstein D, Sultan S, Malhotra J, Santra M, et al. Screening for depression and suicidality in patients with cardiovascular illnesses. Am J Cardiol. 2009;104(9):1194-7.

34. Wong $T$, Xiang $Y$, Tsoh J, Ungvari G, Ko F, Hui D, et al. Suicidal ideation in Chinese patients with chronic obstructive pulmonary disease: a controlled study. Psychogeriatrics. 2016;16(3):172-6.

35. Wang $H$, Wang $M$, Jiang F, Liu Z, Wang H, Li J. Socio-psychological factors relevant to suicidal ideation among patients with AIDS in Changsha. Journal of Central South University (Medical Science). 2017;42(6):687-94 in Chinese.
36. Tang G, Chen Z, Wang Y, Cheng L, Cao Y, Lei J. Current situation and influencing factors of suicidal ideation in patients with gynecological malignancies. Chinese General Practice. 2014;25:3005-8 in Chinese.

37. Inder K, Handley T, Johnston A, Weaver N, Coleman C. Determinants of suicidal ideation and suicide attempts: parallel cross-sectional analyses examining geographical location. BMC Psychiatry. 2014;14:208.

38. Lee Jl, Lee MB, Liao SC, Chang CM, Sung SC, Chiang HC, et al. Prevalence of suicidal ideation and associated risk factors in the general population. Formos Med Assoc. 2010;109:138-47.

39. Martin G, Thomas H, Andrews T, Hasking P, Scott J. Psychotic experiences and psychological distress predict contemporaneous and future nonsuicidal self-injury and suicide attempts in a sample of Australian. Psychol Med. 2015:45:429-37.

40. Mckelvey R, Davies L, Pfaff J, Acres J, Edwards S. Psychological distress and suicidal ideation among 15-24-year-olds presenting to general practice: a pilot study. Australian \& New Zealand Journal of Psychiatry. 1998;32:344.

41. Page R, West J. Suicide ideation and psychosocial distress in sub-Saharan African youth. Am J Health Behav. 2011;35:129-41.

42. Grimsrud A, Dan J, Seedat S, Williams D, Myer L. The association between hypertension and depression and anxiety disorders: results from a nationally representative sample of south African adults. PLoS One. 2009;4:e5552.

43. Scalco A, Scalco M, Azul J, Lotufo N. Hypertension and depression. Clinics. 2005;60:241-50.

44. Mann JJ, Apter A, Bertolote J, Beautrais A, Currier D, Haas A, et al. Suicide prevention strategies: a systematic review. JAMA. 2005;294:2064-74.

45. Wang C. Influence factors analysis of depression in postoperative patients with hypertensive cerebral hemorrhage. Chinese Journal of Practical Nervous Diseases. 2013;16:56-7 in Chinese.

46. Zhu G. Investigation of a psychological problem among hypertension hospitalized patients. For All Health. 2014;1:171.

47. Yu H. Depression condition and the reasoned analysis in the community elderly hypertensive patients. Chinese Journal of Prevention and Control of Chronic Diseases. 2008:15:492 in Chinese.

48. Wu, Y., 2012. Factors associated with suicidal ideation in a rural elderly population of Macheng, Hubei: a matched case-control study, in technology, H.u.o.s.a. (Ed.).

49. Placido A, Sposito A. Association between suicide and cardiovascular disease: time series of 27 years. Int J Cardiol. 2009;135:261-2.

50. Ge D, Sun L, Zhou C, Qian Y, Zhang L, Medina A. Exploring the risk factors of suicidal ideation among the seniors in Shandong, China: a path analysis. J Affect Disord. 2017;207:393-7.

51. Kim, S., Ha, J., Yu, J., Park, D., Ryu, S., 2014. Path analysis of suicide ideation in older people. Int Psychogeriatr, 26 (3): 509-515 26, 509-515.

52. Chen $\mathrm{L}$, Chen $\mathrm{H}$, Zheng M. Association of suicidal ideation and family factors among rural elderly. Chin J Public Health. 2013;29:157-9 in Chinese.

53. Economou M, Madianos M, Peppou L, Theleritis C, Patelakis A. Suicidal ideation and reported suicide attempts in Greece during the economic crisis. World Psychiatry Official Journal of the World. 2013;12:53-9.

54. Fu Z, Feng S, Zhou L, Tang Y, Xiao S. Self-attribution of suicide ideation in a rural sample of Liuyang, Hunan China. J Clin Psychol. 2007b;15:78-80.

55. Gui L, Xiao S. Prevalence and related factors of suicidal ideation and attempts in patients with major depressive episode in rural Community of Liuyang City. Chin Ment Health J. 2009;23:651-5 (in Chinese).

56. Zhang D, Yang $Y$, Wu M, Zhao X, Sun Y, Xie H, et al., 2018. The moderating effect of social support on the relationship between physical health and suicidal thoughts among Chinese rural elderly: a nursing home sample. Int J Ment Health Nurs Jan 23. https://doi.org/10.1111/inm.12436.

57. Yang B, Clum GA. Life stress, social support, and problem-solving skills predictive of depressive symptoms, hopelessness, and suicide ideation in an Asian student population: a test of a model. Suicide Life Threat Behav. 1994:2:127-39.

58. Feng $S$, Xiao S, Zhou L, Tang Y, Fu Z. Risk factors of suicidal ideation in a rural community sample of Hunan. Chin Ment Health J. 2006;20:326-9 (in Chinese).

59. Li C, Tang S. Study on the relationship between suicide ideation and healthpromoting lifestyle in rural elderly. Journal of Hunan Normal University (Medical Sciences). 2016;13:138-41 (in Chinese).

60. Wei T, Zhou L, Yang F. An analysis of 268 cases attempted suicide condition. Chinese Journal of Disease Control \& Prevention. 1999:3:285-6 (in Chinese). 
61. Bruce M, Have TT, Rd R, Katz I, Schulberg H. Reducing suicidal ideation and depressive symptoms in depressed older primary care patients: a

randomized controlled trial. JAMA the Journal of the American Medical Association. 2004;291:1081-91.

\section{Publisher's Note}

Springer Nature remains neutral with regard to jurisdictional claims in published maps and institutional affiliations.

Ready to submit your research? Choose BMC and benefit from:

- fast, convenient online submission

- thorough peer review by experienced researchers in your field

- rapid publication on acceptance

- support for research data, including large and complex data types

- gold Open Access which fosters wider collaboration and increased citations

- maximum visibility for your research: over $100 \mathrm{M}$ website views per year

At $\mathrm{BMC}$, research is always in progress.

Learn more biomedcentral.com/submissions 